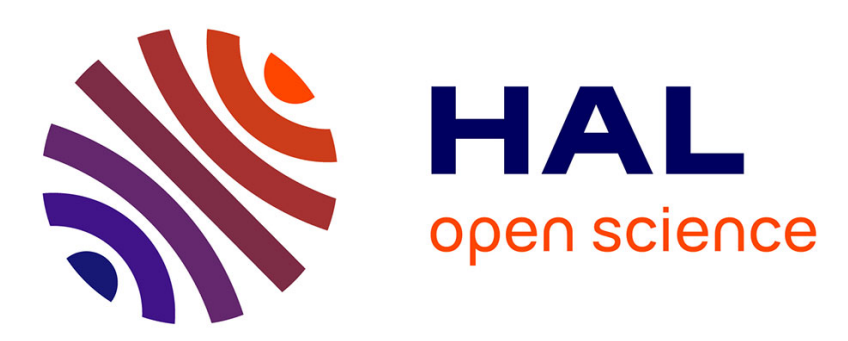

\title{
Study of particle correlation effects on line profiles of Ni-like collisional XUV laser amplifier
}

Annette Calisti, S. Ferri, C. Mossé, B. Talin, A. Klisnick, Limin Meng, D. Benredjem, O. Guilbaud

\section{- To cite this version:}

Annette Calisti, S. Ferri, C. Mossé, B. Talin, A. Klisnick, et al.. Study of particle correlation effects on line profiles of Ni-like collisional XUV laser amplifier. X-Ray Lasers 2012, Jun 2012, Paris, France. pp.49-53, 10.1007/978-3-319-00696-3_2 . hal-00880871

\section{HAL Id: hal-00880871 https://hal.science/hal-00880871}

Submitted on 6 Nov 2013

HAL is a multi-disciplinary open access archive for the deposit and dissemination of scientific research documents, whether they are published or not. The documents may come from teaching and research institutions in France or abroad, or from public or private research centers.
L'archive ouverte pluridisciplinaire HAL, est destinée au dépôt et à la diffusion de documents scientifiques de niveau recherche, publiés ou non, émanant des établissements d'enseignement et de recherche français ou étrangers, des laboratoires publics ou privés. 


\title{
Study of particle correlation effects on line profiles of Ni-like collisional XUV laser amplifier
}

\author{
A. Calisti $\dagger$, S. Ferri $\dagger$, C. Mossé $\dagger$, B. Talin $\dagger$, A. Klisnick $\ddagger$, L. Meng $\ddagger$, D. \\ Benredjem $\S$ and O. Guilbaud $\|$ \\ $\dagger$ PIIM, CNRS-Aix-Marseille Université, Marseille, France \\ $\ddagger$ ISMO, CNRS-Université Paris-Sud, Orsay, France \\ $\S$ LAC, CNRS-Université Paris-Sud, Orsay, France \\ || LPGP, CNRS-Université Paris-Sud, Orsay, France
}

\begin{abstract}
We discuss the effects of particle correlations on the spectral broadening due to the radiator motion (Doppler broadening) for a Ni-like XUV laser line pumped in two different regimes (transient and quasi-steady state regimes) of collisional excitation. In a medium with gain, radiative transport effects modify the observed profile and these modifications depend on the homogeneous or inhomogeneous nature of the intrinsic profile (before amplification). The intrinsic line profile is usually described by a Voigt profile, which is the convolution of a Lorentzian profile due to the different homogeneous broadenings (electron collision-induced transitions and spontaneous emission) and an inhomogeneous Gaussian profile due to Doppler shifts (due to the radiator motion in the free-particle limit). In this paper, it is shown that accounting for the correlations between particles modifies noticeably the radiator-motion broadened profiles, whatever the densities and temperatures values.
\end{abstract}

\section{Introduction}

A better characterization of the spectral properties of existing plasma-based XUV lasers is necessary in order to improve the understanding of some characteristics of these lasers. In particular, the spectral width of the lasing line is an important parameter since it controls the temporal coherence of the source, as well as the shortest duration (Fourier-transform limit) that can be reached for a fully coherent pulse. Moreover, the relative contribution of homogeneous versus inhomogeneous broadening controls the existence of 
re-broadening of the laser linewidth when amplified in the saturation regime. XUV lasers pumped by collisional excitation of Ni-like ions are generated in plasmas with electronic densities and ionic and electronic temperatures very dependent on the parameters of the laser pulse used to induce plasma heating and collisional pumping $\left(N_{e} \sim 5 \times 10^{19}\right.$ to $8 \times 10^{20} \mathrm{~cm}^{-3}, T_{e} \sim 500$ to $1500 \mathrm{eV}$ and $T_{i} \sim 20$ to $500 \mathrm{eV}$ ). For such conditions, plasmas are strongly coupled (plasma coupling parameter, $\Gamma \gg 1$ ) and the ionic interactions have to be taken into account.

In laser-produced plasmas, the main causes of spectral line broadenings are the spontaneous emission, the electronic collisions, the ionic Stark effect and the Doppler frequency shift. It is usual to consider that the spontaneous emission and electronic collisions contribute to the homogeneous broadening and ionic Stark effect and Doppler broadening contribute to inhomogeneous broadening as they are due to local inhomogeneities. Due to the variety of plasma conditions, the relative contributions of the different broadenings are very distinct.

In this article, the PPP line shape code [1] has been used to perform a detailed analysis of the various broadening mechanisms of the spectral profiles of the $4 d-4 p(J=0-1) \mathrm{Ni}$-like Ag lasing line at $\lambda=13.9 \mathrm{~nm}$ for conditions relevant for both transient and quasi-steady state (QSS) regimes of collisional excitation. A study on the accuracy of the free-particle Doppler approximation, versus densities and temperatures has been done by using classical molecular dynamics (MD) simulations.

\section{Spectral line shape modeling}

Including the contribution of ionic emitter motion, the line shape function is given by:

$$
I(\omega)=\Re e \frac{1}{\pi} \int_{0}^{\infty} e^{i \omega t}<e^{i \vec{k} \cdot \vec{r}(t)} \vec{d}(t) \cdot \vec{d}(0)>d t
$$

with $\vec{d}$ the radiator dipole operator and $k=2 \pi / \lambda$.

Ignoring correlations between the ion translation $\vec{r}(t)$ and the dipole moment $\vec{d}(t)$ :

$$
I(\omega)=\Re e \frac{1}{\pi} \int_{0}^{\infty} e^{i \omega t} S_{s}(k, t) C(t) d t
$$

$S_{s}(k, t)=<e^{i \vec{k}} \cdot \vec{r}(t)>$ is the self-structure factor and $C(t)=<\vec{d}(t) \cdot \vec{d}(0)>$ the dipole autocorrelation function. In this work, the PPP line shape code [1] 
is used to calculate $C(t)$. The PPP code is a multi-electron radiator line broadening code developed to calculate theoretical spectral line profiles for a general emitter in a plasma, using data for atomic energy levels and radial matrix elements generated by atomic structure programs [2]. The line profile calculations are done in the framework of the standard theory (quasi-static ion and electron impact approximations) or if necessary including the effects of ionic perturber dynamics by using the Frequency Fluctuation Model [3, 4]. The self-structure factor is well known in the free-particle limit (Doppler effect) resulting from the hypothesis that each radiating ion moves at constant velocity $\vec{r}(t)=\vec{v} t$ with a Maxwellian distribution of velocities, and is given by:

$$
S_{S}(k, t)=e^{-k^{2} t^{2} / 2 \beta m}
$$

with $\beta=1 / k_{B} T$ and $m$ the ion mass. Classical molecular dynamics simulation technique (MD) is a straightforward way to compute $S_{s}(k, t)$ taking into account interactions between ions. The plasma model consists of classical point ions interacting together through a coulombic potential screened by electrons and localized in a cubic box of side $L$ with periodic boundary conditions. Newtons equations of particle motion are integrated by using a velocity-Verlet algorithm using a time-step consistent with energy conservation. Integrating the Newtons equation gives access to the positions and velocities, $\left\{\vec{r}^{N}(t), \vec{v}^{N}(t)\right\}$, of the $N$ ions as a function of time and thus to the associated static and dynamic statistical properties such as structure factors, velocity correlation functions, diffusion coefficients, ion-ion collision rates etc.

\section{Results and discussion}

Spectral line shape calculations have been performed for Ni-like Ag laser $4 d-4 p(J=0-1)$ line at $13.9 \mathrm{~nm}$ for density and temperature ranges chosen to be consistent with population inversion induced by collisional excitation. Two series of results corresponding respectively to transient $\left(T_{i}=20 \mathrm{eV}\right.$, $\left.T_{e}=200 \mathrm{eV}\right)$ and QSS $\left(T_{i}=T_{e}=200 \mathrm{eV}\right)$ pumping regimes, are presented for different electronic densities. Figures 1 and 2 show the full widths at half maximum (FWHM) corresponding to the different mechanisms of broadening taken separately and together as a function of the electronic density for the transient and QSS pumping regimes respectively. It has been checked that the Stark effect associated with ion microfield has a negligible contribution to the line profile. Electronic collisions and spontaneous emission give 


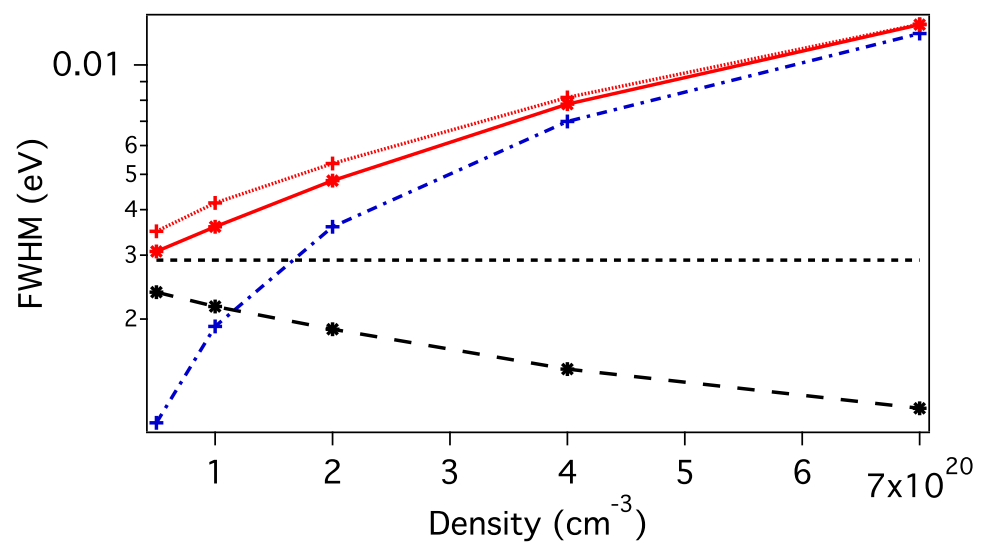

Figure 1. Full width at half maximum versus density of the $4 d-4 p$ laser line in the transient XUV laser case. Broadening due to: - radiator motion in the free particle limit, i.e. Doppler broadening (dashed black line), - accounting for particle interactions (broken black line plus stars), - lifetime broadening (blue chain plus crosses) and total broadening (radiator motion + finite lifetime effects) in the free particle limit (dotted red line plus crosses) and accounting for interactions (full red line plus stars).

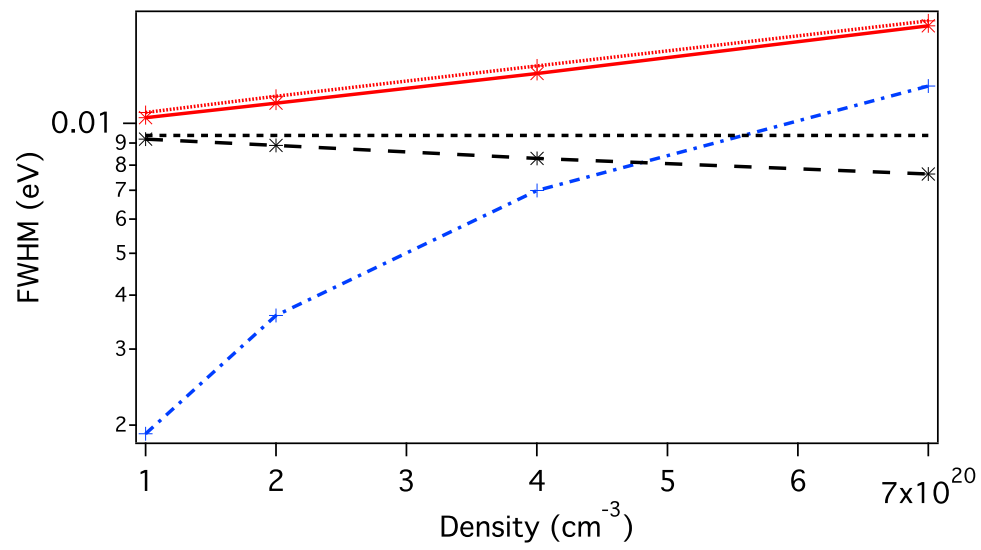

Figure 2. Same as figure 1 in the QSS case. 
rise to the lifetime broadening (blue chain plus crosses) which is homogeneous and increases linearly with $N_{e}$ at a given $T_{e}$. The motion of an emitting ion yields a Doppler shift of the $\mathrm{X}$-ray laser line. If we assume that each radiating ion moves at constant velocity $\vec{r}(t)=\vec{v} t$ with a Maxwellian distribution of velocities, the line profile is inhomogeneous and shows a Gaussian shape with a width (dashed black line) given by a simple analytical formula depending only on $T_{i}$. Accounting for both of the previous effects, the resulting spectral profile will be the convolution of the homogeneous and inhomogeneous profile, leading to the so-called Voigt profile with a width which is a complex combination of both homogeneous and inhomogeneous linewidths (dotted red line plus crosses). Similar results for the spectral profile of the $4 d-4 p(J=0-1)$ lasing line in Ni-like Mo have been obtained and are discussed in the framework of amplification in [5].

In order to account for velocity changing effects, the broadening due to the translational motion of the emitter (broken black line plus stars) has been obtained by using the self-structure factors computed by MD simulations. It can be seen that taking into account interactions between ion emitter and other ions of the plasma gives rise to a narrowing of the profile whatever the considered densities and this narrowing increases with the density. The line profile is no longer Gaussian, so the overall profile is no longer a Voigt profile. Moreover if during their effective lifetimes, the radiating and absorbing ions sample many velocities, not just one as it is supposed in the Doppler free particle limit, the effect of this velocity redistribution will be to homogenize the Doppler component of the intrinsic line profile. Figure 3 shows the evolution in time of the frequency distribution function of a population of radiating emitters chosen to have an initial velocity such as $\vec{k} \cdot \vec{v}(0)=0$. Four different timescales of evolution have been considered, the largest one corresponding to the radiative lifetime (inverse of the lifetime broadening). It can be seen that the velocity redistribution is nearly complete during the radiative lifetime. Similar results have been obtained in the transient pumping regime case.

\section{Conclusion}

This paper concerns a study of the effects of particle correlations on the line broadening due to the radiator motion in the particular case of XUV laser lines. Two different pumping regimes have been considered, transient pumping regime for which ionic temperature is relatively low, so the plasma 


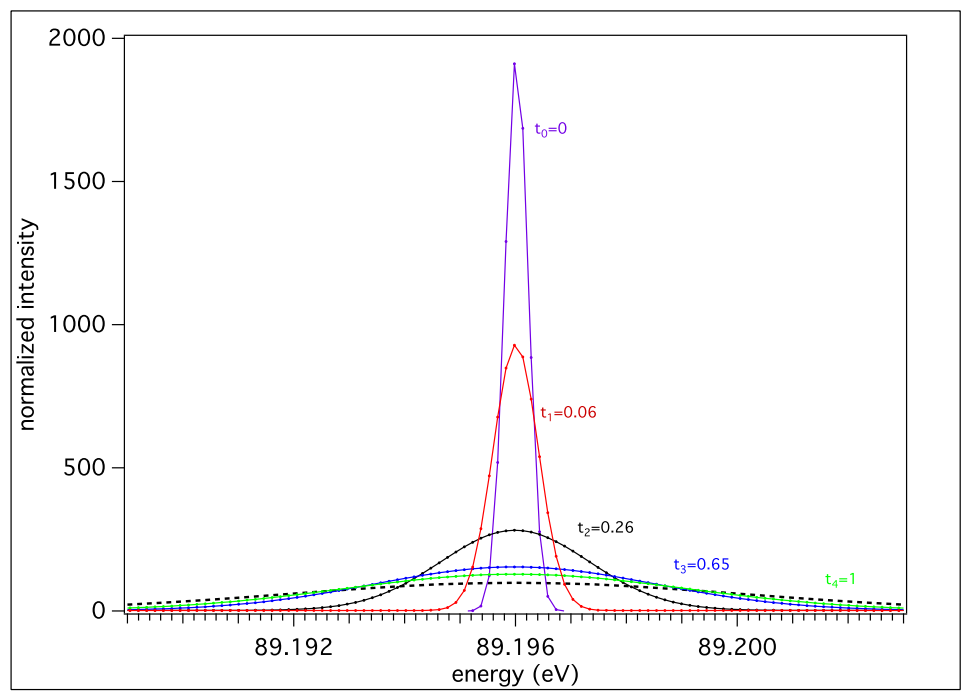

Figure 3. Frequency distribution functions for conditions of the QSS pumping regime at $N_{e}=10^{20} \mathrm{~cm}^{-3}$. Times are in units of radiative lifetimes. The black broken line corresponds to the Doppler free particle limit.

coupling parameter is large, and quasi steady state regime for which the ionic temperature is higher and the plasma coupling parameter of the order of 1 . It has been shown that the effect of correlations cannot be neglected in evaluating Doppler effect for the two cases of interest. For all the considered densities and temperatures, by taking into account correlations between particles, the radiator-motion broadened profiles are modified in being homogenized by velocity redistribution in all the cases and narrowed essentially in the transient regime.

\section{References}

[1] Calisti A. et al 1990 Phys. Rev. A 425433

[2] Grant I. P. et al 1980 Comput. Phys. Commun. 21207

[3] Talin B. et al 1995 Phys. Rev. A 511918

[4] Calisti A. et al 2010 Phys. Rev. E 81016406

[5] Meng L. et al These Proceedings 